This issue has been dealt with in different ways across North Wales and indeed the whole of Wales

Following a review of services in Wrexham during 2017, it was identified that there was an opportunity to pilot a new model which would allocate a designated Consultant to the local Primary Care Mental Health Team (PCMHT)

The Consultant would work entirely within Part 1 of the Mental Health Measure and would offer specialist opinions to Tier 1 Services

Result. PCMHT team members are maintaining open cases for a significant amount of time rather than the $8-10$ sessions that was originally predicted during the implementation of the Mental Health Measures

In order to sustain the service, the minimum number of direct clinical patient contact sessions to be offered by the psychiatrist was up to 4 a week.

During the review period, total number of clinics offered were 51 and a total of 139 patients were offered appointments

Consultants in secondary care covering the same area received exactly 100 less referrals in the first 6 months of the pilot

Main source of referrals to the Tier 1 Consultant came from G.P.'s and the local PCMHT itself

Conclusion. Pilot demonstrated that bringing specialist consultant psychiatrist dedicated to the PCMHT improved the care offered to patients referred by G.P's

Scope of PCMHT needs to extend in order to absorb mild to moderate mental illness and thus avoid patients going into secondary care

This model should be supported, and further resources should be inputted into PCMHT

We should move from a categorical diagnostic referral system to a needs-based intervention where only the most complex cases requiring lengthy interventions shall progress to secondary care

Risk should not be classed as criteria to move patients into secondary care and PCMHT should be able to absorb moderately risky cases

\section{Is pregnancy status being assessed within women's secure services?}

Jeremy Rampling ${ }^{1 \star}$, Shay-Anne Pantall ${ }^{1}$ and Hannah Woodman ${ }^{2}$

${ }^{1}$ Birmingham and Solihull Mental Health NHS Foundation Trust and ${ }^{2}$ University of Birmingham, Medical School

${ }^{\star}$ Corresponding author.

doi: 10.1192/bjo.2021.902

Aims. To establish rates of pregnancy testing on admission of women within a blended secure service.

Background. Women with psychiatric illness are known to be at increased risk of pregnancy, often due to engagement in risky sexual behaviours such as having a higher numbers of sexual partners and engaging in sexual activity whilst under the influence of drugs or alcohol. Awareness of pregnancy at the point of admission to psychiatric hospital would inform ongoing care plans to manage the pregnancy in the safest, least restrictive environment and inform future prescribing decisions, to minimise the risk of teratogenicity associated with some psychotropic medications. Ardenleigh in Birmingham is a blended female secure unit. No pregnancy screening guidelines for this population currently exist. This audit sought to establish current rates of pregnancy testing at the point of admission with a view to developing future guidelines.

Method. A retrospective case note audit of electronic records of all patients admitted to Ardenleigh blended women's service as of 1 st September $2019(n=26)$. The expected standard for pregnancy testing within one month of admission was set as $100 \%$.

Result. Key results include:

The majority of patients (67\%) were aged under 35 years (range 20-56). The most common ethnicities were Caucasian (42\%) and African-Caribbean (38\%). Almost half (46\%) had a primary diagnosis of paranoid schizophrenia.

Two women were known to be pregnant at the point of admission. Only $54 \%$ of women with an unknown pregnancy status were screened for pregnancy within one month of admission.

Rates of screening were particularly poor in women aged under 25 years $(43 \%)$ and between 36 and $45(0 \%)$.

Women not screened for pregnancy were typically admitted from other hospital settings, including AWA services (27\%) or other medium secure units (55\%). 2 women admitted from prison were not tested (29\%)

Of those tested, the majority were checked using urine hCG (92\%).

None of the women tested were found to be pregnant.

Conclusion. Overall pregnancy testing on admission to the unit was poor, with only $54 \%$ of service users screened. Less than $100 \%$ compliance could result in serious consequences for both the woman and unborn baby if a pregnancy is not discovered. Updating the admission checklist for Ardenleigh to include pregnancy testing may prove beneficial. It is recommended that a re-audit is completed 6 months following checklist introduction.

\section{Urine drug screening in women's forensic mental} health services: is current practice meeting guidelines?

Jeremy Rampling ${ }^{1 \star}$, Shay-Anne Pantall ${ }^{1}$ and Ravinder Mann ${ }^{2}$

${ }^{1}$ Birmingham and Solihull Mental Health NHS Foundation Trust and ${ }^{2}$ University of Birmingham, Medical School

${ }^{*}$ Corresponding author.

doi: 10.1192/bjo.2021.903

Aims. To investigate adherence to Trust guidelines for urine drug screening amongst female forensic psychiatric inpatients. Background. The use of illicit substances is an important risk factor which needs to be considered in the management and treatment of forensic psychiatric patients. Research has demonstrated that a high proportion of women admitted within secure services in the UK have a history of substance use. Substance misuse amongst this population can lead to an increased risk of violence, re-offending and mental health relapse; which can pose a significant threat to the safety of other patients, staff and the public. It is therefore important that regular drug screening is carried out to minimise such risks. Ardenleigh is a blended female secure unit in Birmingham. The service has established specific substance use guidelines, outlining the need for each patient to have a personalised drug screen care plan in place. Here we present the findings of an audit completed in 2019.

Method. A six month retrospective electronic case note audit for female inpatients admitted to Ardenleigh as of 1st September $2019(\mathrm{n}=27)$. We compared drug screen care plans and frequency of urine drug screens over 6 months with the recommendations of the current service-specific Trust guidelines. Care plans should include: information regarding random drug screening; frequency of random drug screening; triggers for increased risk of substance misuse; and consequences for a positive test result to be contained within inpatient care plans.

Result. Patient aged between 20 and 56 years old (median age 31 ). Fewer than half of inpatients $(41 \%)$ had a documented random 